\title{
Updated Perspectives on the Management of Relapsed and Refractory Multiple Myeloma
}

\author{
Linda Heimberg Stefan Knop \\ Medizinische Klinik II, Universitätsklinikum Würzburg, Würzburg, Germany
}

\section{Keywords}

Myeloma · Lenalidomide-refractory patients

\begin{abstract}
Background: With the availability of T-cell-directed therapy and next-generation compounds of established classes of drugs, the treatment of relapsed/refractory $(r / r)$ myeloma is getting more complex. However, treatment options in practice are limited by availability, approval, and patient comorbidity. The aim of this article is to provide a practical approach toward the choice of treatment for $r / r$ myeloma patients. Summary: Regarding market authorization and current guidelines, at least in Germany, most patients nowadays will have received a doublet or triplet combination as first-line therapy containing a proteasome inhibitor and an immunomodulatory drug, mostly lenalidomide. We focus on the treatment options for patients that are ineligible for (another) stem cell transplantation. We will review treatment options for relapse after first- or second-line therapy and beyond third-line. Key Messages: There is promising data supporting the efficacy and safety of triplet combinations containing anti-CD38-monoclonal antibodies (anti-CD38 mAbs) at first or second relapse in combination with next-generation compounds. For the treatment beyond third-line, comparative studies are scarce but some promising compounds are available via conditional authorization, and there is more to come in the future. We will present some early phase trials featuring promising results. @ 2021 The Author(s).

Published by S. Karger AG, Basel
\end{abstract}

\section{Introduction}

ESMO guidelines offer an abundance of options and differ in various scenarios, but still 9 options listed in the relapsed/refractory $(\mathrm{r} / \mathrm{r})$ setting for "bortezomib-sensitive" patients after VRd looks not very helpful for the individual case [1]. We will try to describe existing treatments and stratify or give a certain order to choose from considering comorbidities and availability.

\section{When to Start Treatment?}

The decision whether to start treatment at biochemical disease progression or with the occurrence of symptoms is not always straightforward. By far the most patients eventually develop a clinical relapse. There is some data pointing toward a PFS and OS benefit for early treatment $[2,3]$. Presumably, this holds true especially for high-risk myeloma. The situation should be discussed with the patient individually considering cytogenetic risk, aggressiveness of the biochemical relapse, response, and treatment tolerability at earlier lines and comorbidity [4].

\section{Diagnostic Procedures at the Time of Progression}

It is generally recommended to repeat all diagnostic procedures that were abnormal at the initial staging and to add different imaging modalities only when new disease manifestations are clinically suspected, for example,

\section{karger@karger.com} www.karger.com/ort

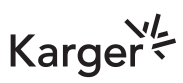

bOPEN ACCESS
(C) 2021 The Author(s)

Published by S. Karger AG, Basel

This article is licensed under the Creative Commons Attribution 4.0 International License (CC BY) (http://www.karger.com/Services/ OpenAccessLicense). Usage, derivative works and distribution are permitted provided that proper credit is given to the author and the original publisher.
Correspondence to:

Stefan Knop, knop_s@ukw.de 
MRI for extramedullary disease. Whole-body low-dose $\mathrm{CT}$ is much more sensitive than conventional $\mathrm{X}$-ray. Therefore, it should be part of the initial staging whenever possible. MRI is most sensitive in the detection of focal intramedullary lesions and extraosseous disease and should be used additionally in patients with a negative CT scan [5]. Its widespread use is limited by availability, high costs, and patient factors (claustrophobia, inability to lie still).

PET-CT scan can replace conventional CT scan as long as it fulfills all criteria for conventional CT skeletal survey. However, in Germany, reimbursement of costs for PET-CT-scanning in myeloma patients is restricted. An advantage of the PET-CT during follow-up is that it may detect focal bone marrow infiltration in cases of sampling error with false-negative bone marrow aspirate and that it may differentiate active disease manifestations from inactive lesions or differential diagnosis such as scars. However, bone marrow enhancement can be false positive during recovery phase and osteolytic activity can be confused with bone healing processes [5].

When progress is suspected it is reasonable to first repeat low-dose CT scanning that can be compared to the initial staging. If this remains negative and there is serological progression or clinical signs of disease activity (e.g., suspected extramedullary manifestation), an MRI or a PET-CT scan can be added. However, in the absence of serological progression, it is recommended to obtain adequate histology of suspected extramedullary lesions, since treatment changes should not be based on imaging alone [5].

Concerning repeat bone marrow aspiration all patients should at least have a baseline sample sent for fluorescence in situ hybridizytion to detect IgH translocations (i.e., $t[4 ; 14]$ and $t[11 ; 14])$, which are early events, and secondary events that are high-risk features such as del17p and 1q21 gains. Fluorescence in situ hybridizytion testing should possibly be repeated at relapse (if not high risk in the first place) and include analysis for del17p, ampl/gain $1 \mathrm{q}$ and $t(11 ; 14)$. Del17p is often acquired during disease course and conveys an unfavorable prognosis. There are not yet specific recommendations for high-risk patients in the relapsed setting. However, the knowledge of high-risk cytogenetics might influence treatment decisions such as when to start treatment, intensity of the treatment regimen, and indication for transplantation [6].

There are some examples that molecularly targeted treatments may be particularly useful, such as Venetoclax in patients with $t(11 ; 14)$. Venetoclax as a late-line therapeutic option will be discussed at the end of this review. About $4 \%$ of myeloma patients carry an activating BRAFmutation at diagnosis, the proportion doubles (app. 8\%) at relapse [7]. It has no prognostic impact so far. How- ever, results from the BIRMA trial indicate that the combination of the BRAF-inhibitor encorafenib and the MEK-inhibitor binimetinib might be effective in the treatment of BRAF-mutated myeloma, with an ORR of $82 \%$ in a small patient collective. There is some experience with the combination of a BRAF-inhibitor and a MEK-inhibitor in the treatment of solid tumors and the side effects observed in myeloma patients did not differ from those reported in solid tumors [7]. Thus, targeted mutational analysis at the time of progression might become even more important in the future.

Bone marrow aspiration is not required prior to immune-therapies, neither for anti-CD38 nor for BCMAdirected therapies. When anti-CD38 antibody-retreatment is considered, it may be useful to reassure that there is still CD38+-expression on plasma cells, since there are case reports of CD38-loss at relapse $[8,9]$.

\section{Can "Next Generation Compounds" Overcome Resistance?}

Since lenalidomide is commonly used in first-line therapy and often combined with bortezomib, a high number of patients are pretreated with both substances early in their treatment course. Is lenalidomide retreatment feasible? Patients that progressed are usually excluded from further trials using the same compound, whereas in real-world retreatment or treatment beyond progression is not exceptional especially as part of a combination therapy. Treatment tolerability as well as aggressiveness of the progression should be taken into consideration [10].

\section{Is Pomalidomide an Option for Lenalidomide- Refractory Patients?}

While all IMiDs (ImmunomodulatoryDrugs) exert their effects by targeting cereblon, pomalidomide has a distinct pharmacology compared to lenalidomide and, despite having a comparable binding affinity, results in higher efficacy by faster degradation of its substrates [11, 12]. It can induce responses in lenalidomide-refractory patients in combination with low-dose dexamethasone (ORR $32-33 \%[11,13]$ ), even directly after failure of lenalidomide and especially in patients who received only low-dose lenalidomide (15 mg or less) [12]. Pomalidomide is approved in Europe for second-line therapy in combination with bortezomib and dexamethasone ( $\mathrm{Vd})$ as well as in third-line therapy in combination with dexamethasone or $\mathrm{Vd}$ in patients pretreated with lenalidomide [14]. 


\section{Is Carfilzomib an Option for Bortezomib-Refractory Patients?}

Proteasome inhibitors induce plasma cell apoptosis by blocking protein degradation. Carfilzomib is an irreversible proteasome inhibitor in contrast to bortezomib and ixazomib, which are reversible proteasome inhibitors [15]. The ENDEAVOR trial compared carfilzomib/dexamethasone to bortezomib/dexamethasone and included patients who were pretreated with bortezomib. In direct comparison, carfilzomib showed higher response rates (ORR 77\% vs. 63\%) and an overall survival benefit of 7 months than bortezomib (47 months vs. 40 months) [16]. One important toxicity of bortezomib is peripheral neuropathy. This side effect occurs infrequently following carfilzomib and patients with preexisting neuropathy can be treated with carfilzomib without worsening of neuropathic symptoms [17]. Carfilzomib can cause hypertension and cardiac failure as well as renal failure. Thus, it should be used with caution in patients with preexisting cardiac constraint and patients with preexisting hypertension [18]. Ixazomib was not effective in a small number of bortezomib-refractory patients (ORR/CBR $=0 \%$ for $3 \mathrm{mg}$ and $18.7 \%$ for $4 \mathrm{mg}$ ). Adverse events were mostly hematotoxic [19].

\section{Anti-CD38-Monoclonal Antibodies}

Daratumumab is a monoclonal antibody targeting CD38 (anti-CD38 mAb). It exerts its cytotoxic effects by recruitment and activation of immune cells and the complement system. It is effective as monotherapy in $\mathrm{r} / \mathrm{r}$ myeloma and has a favorable toxicity profile. An ORR of $29 \%$ in heavily pretreated patients was observed in the SIRIUS trial [20]. Isatuximab is an anti-CD38 $\mathrm{mAb}$ that can additionally induce apoptosis in CD38+-cells directly independent of other immune cells. To the best of our knowledge, isatuximab has not been directly compared to daratumumab yet. The data for isatuximab used in subjects with daratumumab pretreatment is scarce and limited to heavily pretreated patients. It suggests that a longer interval after daratumumab without anti-CD38 treatment increases the likelihood of response to isatuximab [21].

Regarding the current standard in Europe, most patients receive at least lenalidomide or bortezomib in first-line therapy and many patients receive both substances upfront. Treatment options to consider at first relapse after VRD are daratumumab/pomalidomide/ dexamethasone (DPd), anti-CD38 mAb/carfilzomib/ dexamethasone (DKd or IsaKd) or, for patients that are unable to tolerate a more intensive regime, daratumumab monotherapy. For patients who are not bortezomib- refractory, DVd and PVd are additional options. Elotuzumab (Elo)/pomalidomide/dexamethasone has received EMA approval from third-line therapy and onward in 2019 [1].

For patients considered lenalidomide-sensitive, KRd, DaraRd and Elotuzumab/lenalidomide/dexamethasone or IxaRd would be additional options. Here, we will focus on lenalidomide-refractory patients [1].

In the OPTIMISMM trial, PVd was compared to Vd in $\mathrm{r} / \mathrm{r}$ myeloma. All patients had pretreatment with lenalidomide, and a large subgroup was lenalidomide-refractory (60\%). There was a mPFS benefit of about 4 months (11.2 vs. 7.1 months; HR 0.61) for all patients including the subgroup of lenalidomide-refractory patients ( 9.5 vs. 5.6 months). It must be pointed out that $59 \%$ of the patients with only 1 prior line of therapy had pretreatment with bortezomib [22]. Thus, even if bortezomib-refractory patients were excluded from the trial, in a real-world situation $\mathrm{Vd}$ would not be considered an optimal treatment option for the patients in the control arm.

Daratumumab-Pd (DPd) was compared to Pd in the APOLLO trial. All patients had at least 1 prior line of therapy including pretreatment with lenalidomide and $80 \%$ of patients were lenalidomide-refractory. DPd resulted in a mPFS improvement of 5.5 months ( 12.4 vs. 6.9 months; HR 0.63) [23].

The ICARIA trial-tested isatuximab-Pd versus $\mathrm{Pd}$ in patients that were pretreated with a medium of 3 prior lines and were nonresponsive or refractory or could not tolerate lenalidomide, with a high proportion of patients being both refractory to lenalidomide and a proteasome inhibitor (71\%). Isatuximab-Pd resulted in an $\mathrm{mPFS}$ benefit of 5 months ( 11.5 vs. 6.5 months; HR 0.6$)$ and was adequately tolerated [24]. Of note, in these 2 latter studies, patients randomized to Pd apparently fared better (in terms of a cross-trial PFS comparison) in contrast to the pivotal study [25].

$\mathrm{Kd}$ seems to be an option as a doublet in lenalidomiderefractory patients with modest PFS of about 8 months $[16,26]$. In the CANDOR trial, daratumumab-Kd (DKd) was tested against $\mathrm{Kd}$ in $\mathrm{r} / \mathrm{r}$ myeloma patients with a median of 2 prior therapy lines and lenalidomide refractoriness in 33\% of patients. Treatment with DKd resulted in an increase in $\mathrm{mPFS}$. MPFS was not reached for DKd versus 15.8 months for Kd (HR 0.63) [27], an update was presented at ASH2020 with an mPFS of 28.6 months in the DKd-arm versus 15.6 months in the Kd arm (HR 0.59) [28]. The mPFS benefit was maintained in the group of lenalidomide-refractory patients (HR 0.45). The proportion of patients who discontinued treatment due to adverse events was approximately the same in both treatment arms (22\% vs. $25 \%)$. Bronchial infection was more common in the daratumumab arm [27]. 
Table 1. Overview: treatment options for lenalidomide-refractory patients at first or second relapse

\begin{tabular}{llllll}
\hline Treatment & Landmark study & $\begin{array}{l}\text { Median prior } \\
\text { lines of therapy }\end{array}$ & $\begin{array}{l}\text { \% Lenalidomide- } \\
\text { refractory patients }\end{array}$ & mPFS & EMA approval \\
\hline DKd versus Kd & CANDOR $n=466[27,28]$ & 2 & 33 & 28.6 versus 15.6 months (HR 0.59) & $\begin{array}{l}\text { Approved (second line and } \\
\text { beyond) }\end{array}$ \\
\hline IsaKd versus Kd & IKEMA $n=302[29]$ & 2 & 33 & $\begin{array}{l}\text { Not reached versus } 19.1 \text { months } \\
\text { (HR 0.53) }\end{array}$ & $\begin{array}{l}\text { Approved (second line and } \\
\text { beyond) }\end{array}$ \\
\hline DPd versus Pd & APOLLO $n=304[23]$ & 2 & 80 & 12.4 versus 6.9 months (HR 0.63) & $\begin{array}{l}\text { Approved (len-refractory, third } \\
\text { line and beyond) }\end{array}$ \\
\hline IsaPd versus Pd & ICARIA $n=308$ [24] & 3 & 93 & 11.5 versus 6.5 months & Approved (third line and beyond) \\
\hline EloPd versus Pd & ELOQUENT 3 & 3 & 87 & 10.3 versus 4.7 months & Approved (third line and beyond) \\
\hline DVd versus Vd & CASTOR [30] & 2 & 21 & 16.7 versus 7.1 months (HR 0.31) & $\begin{array}{l}\text { Approved (second line and } \\
\text { beyond) }\end{array}$ \\
\hline PVd versus Vd & OPTIMISMM $n=559[22]$ & 2 & 60 & 11.2 versus 7.1 months (HR 0.61) & $\begin{array}{l}\text { Approved (second line and } \\
\text { beyond) }\end{array}$
\end{tabular}

Dpd, daratumumab/pomalidomide/dexamethasone; EloPd, Elotuzumab (Elo)/pomalidomide/dexamethasone; DKd, daratumumab-Kd; IsaKd, isatuximab-Kd; IsaPd, isatuximab-Pd.

The combination isatuximab-Kd (IsaKd) was compared to Kd in $\mathrm{r} / \mathrm{r}$ myeloma in the IKEMA trial. Patients had a median of 2 prior therapy lines and approximately $33 \%$ of patients were refractory to lenalidomide. An mPFS benefit was observed favoring IsaKd (mPFS not reached at 20.7 months' follow-up for IsaKd vs. 19.1 months for $\mathrm{Kd}$; HR 0.53). The proportion of patients who discontinued treatment due to adverse events was lower in the IsaKd arm (8.5\% vs. 13.9\%). Pneumonia and dyspnea were more frequently reported in the isatuximab arm [29]. Study characteristics and outcomes are displayed in Table 1.

In conclusion, after failing first-line therapy, a triplet combination should be preferred over a doublet. AntiCD38 mAbs obviously add benefit to next-generation Imids or PIs with good tolerability. We need more data concerning daratumumab-refractory patients.

The mPFS rates reported in the anti-CD38 $\mathrm{mAb}-\mathrm{Pd}$ trials $[23,24]$, appear to be shorter than mPFS in the anti-CD38 mAb-Kd trials $[28,29]$, but those cannot be compared due to the differences in trial design and patient populations. The proportion of lenalidomide-refractory patients was higher in the APOLLO trial and especially high in the ICARIA trial where patients were more advanced in their disease course $[23,24]$. Treatment selection should be based on comorbidity and prior therapy. We need more studies to compare the triplets with each other. Of note, carfilzomib-based mAb combinations are intended to be "continuous" rather than "fixed duration" treatments as a major discrepancy between carfilzomib/dex and bortezomib/dex "backbones" $[28,30]$. This might, at least in part account for their excellent efficacy.

\section{Beyond Third-Line Therapy}

Patients that are refractory to $2 \mathrm{IMiDs}, 2$ proteasome inhibitors and daratumumab, are called "penta-refractory" [31]. They pose a challenge to their treating physicians and should preferably be treated in clinical trials if available. Fortunately, some new treatment options have come up for this patient population. It goes beyond the scope of this review to list all of them. We chose to mention some of them in more detail. We will conclude with an outlook to promising treatment options that are currently in early clinical trial phase.

Belantamab mafodotin is the first-in-class antibodydrug conjugate directed against BCMA. It has been granted conditional authorization in the EU based on the DREAMM-2 study [32] that compared 2 different dose levels of belantamab mafodotin $(2.5 \mathrm{mg} / \mathrm{kg}$ vs. $3.4 \mathrm{mg} / \mathrm{kg})$ with each other but did not compare the substance to standard of care anti-myeloma treatment. The trial was conducted in heavily pretreated (at least triple-refractory) patients with a median of 6-7 prior lines of therapy and resulted in an ORR of $31 \%(2.5 \mathrm{mg} / \mathrm{kg})$ versus $34 \%$ $(3.4 \mathrm{mg} / \mathrm{kg})$ and a mPFS of 2.8 months $(2.5 \mathrm{mg} / \mathrm{kg})$ versus 3.9 months $(3.4 \mathrm{mg} / \mathrm{kg})$. However, all patients experienced adverse events [33]. Even at the lower dose level of $2.5 \mathrm{mg} / \mathrm{kg}$, grade $3 / 4$ adverse events occurred in $83 \%$ of the patients [32]. The most frequently observed grade $3 / 4$ adverse events were keratopathy (27\%) and hematotoxicity (thrombocytopenia 20\% and anemia 20\%). Eight percent of the patients treated with $2.5 \mathrm{mg} / \mathrm{kg}$ discontinued treatment because of side effects, mostly due to keratopathy [33]. Comparative trials to established myeloma treatment regimes, such as $\mathrm{Pd}$, are already underway 
Table 2. Selected treatment options beyond third-line therapy

\begin{tabular}{llllll}
\hline Treatment & Landmark study & $\begin{array}{l}\text { Median prior } \\
\text { lines of therapy }\end{array}$ & ORR, \% & mPFS & EMA approval \\
\hline Belamaf $2.5 \mathrm{mg} / \mathrm{kg}$ vs. 3.4 mg/kg & DREAMM $2 n=196[33]$ & $6-7$ & 31 vs. 34 & 2.8 vs. 3.9 months & Conditional authorization \\
Selinexor & STORM $N=122[34]$ & 7 & 26 & 3.7 months & Conditional authorization \\
VenetoclaxVd vs. PlaceboVd & BELLINI $n=291[36]$ & $1-3$ & 82 & 22.4 vs. 11.5 months & Orphan designation \\
Melflufen & HORIZON $n=157[38]$ & 5 & 30 & 4.2 months & Not yet approved \\
Idecabtagene vicleucel & KarMMa $n=128[42]$ & 6 & 73 & 8.6 months & $\begin{array}{l}\text { Positive EMA CHMP opinion (Jun } \\
\end{array}$ \\
& & & & & 2021) \\
\hline
\end{tabular}

Belamaf, belantamab mafodotin; Melflufen, Melphalan flufenamide.

(DREAMM 3) [32]. Patients should be carefully selected with regard to the hematotoxic side effects.

Selinexor is an inhibitor of exportin 1 thereby blocking nuclear export. It has been evaluated in the STORM trial in combination with dexamethasone in patients that were pretreated with a median of 7 prior therapy lines. The investigators observed an ORR of $26 \%$ and a mPFS of 3.7 months. Severe thrombocytopenia was the most common grade $3 / 4$ toxicity and occurred in $59 \%$ of patients. Eighteen percentage of the patients discontinued treatment because of side effects [34]. Again, the EMA granted conditional authorization because of a favorable risk-tobenefit ratio [35].

Venetoclax is the first example of personalized therapy based on molecular cytogenetic testing in myeloma. In the BELLINI trial, it was shown that venetoclax in combination with bortezomib/dexamethasone improves progression-free survival by about 9 months compared to Vd. However, there were more treatment-related fatal infections so that venetoclax in combination with bortezo$\mathrm{mib} /$ dexamethasone had an unfavorable risk-to-benefit ratio except for patients with chromosomal aberration $t$ $(11 ; 14)$ and high bcl2-expression for whom PFS benefit was more pronounced but failed to demonstrate an OS advantage. In fact, regarding the increased risk of neutropenia and infection [36] patients should be carefully selected for venetoclax treatment. Other molecularly targeted therapies are under development.

Iberdomide is a modulator of cereblon-E-ligase that targets cereblon in a different way compared to the other IMiDs. So far, only very few results were reported. It is tested in 2 triplet combinations, iberdomide/daratumumab/dexamethasone (IberDd) versus iberdomide/ bortezomib/dexamethasone (IberVd) in a small group of heavily pretreated patients (19 patients with a median number of 4 lines of therapy, ranging from 1 to 12 lines, vs. 21 patients with a median number of 6 prior lines of therapy, ranging from 1 to 14 lines). As far as the results are comparable in such small groups of patients, an ORR of 35\% (IberDd) versus 50\% (IberVd) was observed. This would be in line with the treatment options mentioned earlier. The proportion of patients experiencing grade $3 / 4$ events was 78\% (IberDd) versus 65\% (IberVd). Toxicity was mostly hematotoxic [37]. So far, there is no comparison of iberdomide combinations to established triplets, but we expect to learn more about this substance and its therapeutic potential in the future.

Melphalan flufenamide (Melflufen) is a peptide-conjugated lipohilic alkylating agent for intravenous injection. It was tested in the HORIZON trial in combination with dexamethasone in pretreated patients (medium number of 5 prior lines) with a relatively high proportion of extramedullary disease (35\% of the patients included). An ORR of $30 \%$ with a mPFS of 4.2 months was reported. However, $96 \%$ of patients experienced grade $3 / 4$ adverse events that were mostly hematotoxic. Seventy-nine percentage of patients developed grade $3 / 4$ neutropenia, $76 \%$ grade $3 / 4$ thrombocytopenia, and $43 \%$ grade $3 / 4$ anemia. It deserves special mentioning that $24 \%$ of the patients with extramedullary disease had at least a partial remission [38], what seems promising in this difficult-to-treat patient population [39]. An overview of recent study results is displayed in Table 2.

Numerous studies tested anti-BCMA CAR-T-cell therapy in very small subsets of heavily pretreated $\mathrm{r} / \mathrm{r}$ myeloma patients with impressive response rates over short observational periods [40]. One of the larger studies utilizing the second-generation compound idecabtagene vicleucel was reported by Raje et al. [41] with an ORR of $85 \%$ in 33 patients with a median number of 7 prior lines of therapy. Thirty-six patients were enrolled in the study, but 3 patients ( $8 \%$ ) did not make it to CAR-T-cell reinfusion because of progression after leukapheresis. Ninetyseven percentage of patients experienced grade $3 / 4$ adverse events that were mostly hematotoxic ( $85 \%$ of patients developed grade $3 / 4$ neutropenia, $45 \%$ of patients developed grade $3 / 4$ thrombocytopenia) and attributable to the lymphodepleting chemotherapy. The incidence of grade 3 CRS was low (6\%), and there was no grade 4 CRS. One grade 4 neurologic event was observed [41]. The KarMMa trials are about to test BCMA-directed CAR-Tcells in larger patient cohorts. First results from the 
KarMMa trial were presented at the ASCO 2020. In 128 patients, pretreated with a medium number of 6 prior lines of therapy, ORR was $73 \%$. The toxicity observed was again mostly hematotoxicity. Grade 3 CRS was observed in $5 \%$ of patients and grade $3 / 4$ neurologic events were observed in $3 \%$ of patients [42]. It remains to be seen how durable responses are.

One difficulty about CAR-T-cell therapy is to get the patient through the time between apheresis and CAR-Tcell infusion. In contrast, bispecific antibodies are offthe-shelf products. Early clinical data about the bispecific antibody talquetamab, CD3 $\times$ GPCR5, look very promising. The ORR was $63 \%$ in heavily pretreated patients (median prior lines of therapy: 5.5 ). The most important grade $3 / 4$ events reported were neutropenia (45\%), anemia (29\%), and CRS (4\%). No grade 3/4 neurotoxicity occurred. Most frequent low-grade AEs (75\%, no grade 3/4) were skin related [43]. Teclistamab is another bispecific antibody, CD $3 \times$ BCMA. It resulted in an ORR of $63.8 \%$ in heavily pretreated patients (median number of 6 prior lines of therapy). Grade 3/4 events, observed in $39 \%$ of the patients, were mostly neutropenia (23\%), infection-related AEs (15\%), and anemia (9\%). No grade 3/4 CRS occurred. Grade 3/4 neurotoxicity occurred in $2 \%$ of the patients [44]. The results for the bispecific antibodies, with some of them even administered via subcutaneous injection $[43,44]$, seem very promising and leave us excited about further clinical data.

\section{Conclusion}

In conclusion, there are several new treatment options for patients with $\mathrm{r} / \mathrm{r}$ myeloma. The anti-CD38 mAb-triplets with carfilzomib or pomalidomide are effective and safe options at first or second relapse including in lenalidomide-refractory patients. Beyond third-line therapy, several new treatment options have emerged and are currently further investigated. Some of them are already available via conditional authorization. Since we might miss patients with each line of therapy, we need more comparative trials and real-world data to make the treatment choices based on an individual risk-to-benefit assessment.

\section{Conflict of Interest Statement}

L.H. has no conflicts of interest to declare. S.K. received honoraria from Amgen, BMS, Celgene, Novartis, oncopeptides, and Sanofi. He received research funding from Amgen, BMS, and Celgene.

\section{Funding Sources}

The authors received no funding for conception or writing of this article.

\section{Author Contributions}

Both authors have contributed equally.

\section{References}

1 Dimopoulos MA, Moreau P, Terpos E, Mateos MV, Zweegman S, Cook G, et al. Multiple myeloma: EHA-ESMO clinical practice guidelines for diagnosis, treatment and follow-up. Ann Oncol. 2021;32(3):309-22.

2 Katodritou E, Kyrtsonis MC, Delimpasi S, Kyriakou D, Symeonidis A, Spanoudakis E, et al. Real-world data on Len/Dex combination at second-line therapy of multiple myeloma: treatment at biochemical relapse is a significant prognostic factor for progression-free survival. Ann Hematol. 2018;97:1671-82.

3 Moreau P, Siegel DS, Goldschmidt H, Niesvizky R, Bringhen S, Orlowski RZ, et al. Subgroup analysis of patients with biochemical or symptomatic relapse at the time of enrollment in the endeavor study. Blood. 2018;132(Suppl 1):3243.

4 Ludwig H, Sonneveld P, Davies F, Bladé J, Boccadoro M, Cavo M, et al. European perspective on multiple myeloma treatment strategies in 2014. Oncologist. 2014;19(8): 829-44.

5 Hillengass J, Usmani S, Rajkumar SV, Durie BGM, Mateos MV, Lonial S, et al. International myeloma working group consensus recommendations on imaging in monoclonal plasma cell disorders. Lancet Oncol. 2019; 20(6):e302-12.
6 Caro J, Al Hadidi S, Usmani S, Yee AJ, Raje N, Davies FE. How to treat high-risk myeloma at diagnosis and relapse. Am Soc Clin Oncol Educ Book. 2021;41:291-309.

7 Raab MS, Giesen N, Scheid C, Besemer B, Miah K, Benner A, et al. Safety and preliminary efficacy results from a phase II study evaluating combined BRAF and MEK inhibition in relapsed/refractory multiple myeloma (rrMM) patients with activating BRAF V600E mutations: the GMMG-birma trial. Blood. 2020;136(Suppl 1):44-5.

8 Hotchandani N, Fung H, Dulaimi E. CD38 expression loss in multiple myeloma treated with daratumumab. Am J Clin Pathol. 2016; 146(Suppl_1):S62-81

9 Minarik J, Novak M, Flodr P, Balcarkova J, Mlynarcikova M, Krhovska P, et al. CD38negative relapse in multiple myeloma after daratumumab-based chemotherapy. Eur J Haematol. 2017;99:186-9.

10 Kunacheewa C, Feng L, Manasanch EE, Bashir Q, Patel KK, Mehta R, et al. Outcomes of lenalidomide retreatment with novel triplet regimens in patients with multiple myeloma progressing on lenalidomide-based maintenance therapy. Br J Haematol. 2021;193(3): e23-6.
11 Siegel DS, Schiller GJ, Song KW, Agajanian R, Stockerl-Goldstein K, Kaya H, et al. Pomalidomide plus low-dose dexamethasone in relapsed refractory multiple myeloma after lenalidomide treatment failure. Br J Haematol. 2020;188:501-10.

12 Bjorklund CC, Lu L, Kang J, Hagner PR, Havens CG, Amatangelo $M$, et al. Rate of CRL4(CRBN) substrate Ikaros and Aiolos degradation underlies differential activity of lenalidomide and pomalidomide in multiple myeloma cells by regulation of c-Myc and IRF4. Blood Cancer J. 2015 Oct 2;5(10):e354.

13 Richardson PG, Siegel DS, Vij R, Hofmeister CC, Baz R, Jagannath S, et al. Pomalidomide alone or in combination with low-dose dexamethasone in relapsed and refractory multiple myeloma: a randomized phase 2 study. Blood. 2014;123(12):1826-32.

14 EMA. Imnovid EPAR product information. 2019 [cited 2021 Aug 10]. Available from: https: //www.ema.europa.eu/en/medicines/ human/EPAR/imnovid.

15 Chim CS, Kumar SK, Orlowski RZ, Cook G, Richardson PG, Gertz MA, et al. Management of relapsed and refractory multiple myeloma: novel agents, antibodies, immunotherapies and beyond. Leukemia. 2018;32:252-62. 
16 Dimopoulos MA, Goldschmidt H, Niesvizky R, Joshua D, Chng WJ, Oriol A, et al. Carfilzomib or bortezomib in relapsed or refractory multiple myeloma (ENDEAVOR): an interim overall survival analysis of an open-label, randomised, phase 3 trial. Lancet Oncol. 2017; 18(10):1327-37.

17 Niesvizky R, Hungria V, Ho J, Suvorov A White D, Ben-Yehuda D, et al. Rates of peripheral neuropathy $(\mathrm{PN})$ in patients $(\mathrm{Pts})$ with relapsed and refractory multiple myeloma (RRMM) treated with carfilzomib vs comparators in pivotal phase III trials. J Clin Oncol. 2017;35(15_Suppl):8041.

18 Astarita A, Mingrone G, Airale L, Vallelonga F, Covella M, Catarinella C, et al. Multiple myeloma patients undergoing carfilzomib: development and validation of a risk score for cardiovascular adverse events prediction. Cancers. 2021;13(7):1631

19 Berenson JR, Cohen A, Spektor TM, Bitran JD, Chen GQ, Moezi MM, et al. Replacement of ixazomib for relapsed/refractory multiple myeloma patients refractory to a bortezomib or carfilzomib-containing combination therapy. J Clin Oncol. 2017;35(15_Suppl):8013.

20 Lonial S, Weiss BM, Usmani SZ, Singhal S, Chari A, Bahlis NJ, et al. Daratumumab monotherapy in patients with treatment-refractory multiple myeloma (SIRIUS): an open-label, randomised, phase 2 trial. Lancet. 2016;387(10027):1551-60.

21 Mikhael J, Belhadj-Merzoug K, Hulin C, Vincent L, Moreau P, Gasparetto C, et al. A phase 2 study of isatuximab monotherapy in patients with multiple myeloma who are refractory to daratumumab. Blood Cancer J. 2021; 11(5):89.

22 Dimopoulos M, Weisel K, Moreau P, Anderson LD, White D, San-Miguel J, et al. Pomalidomide, bortezomib, and dexamethasone for multiple myeloma previously treated with lenalidomide (OPTIMISMM): outcomes by prior treatment at first relapse. Leukemia. 2021;35(6):1722-31.

23 Dimopoulos MA, Terpos E, Boccadoro M, Delimpasi S, Beksac M, Katodritou E, et al. Daratumumab plus pomalidomide and dexamethasone versus pomalidomide and dexamethasone alone in previously treated multiple myeloma (APOLLO): an open-label, randomised, phase 3 trial. Lancet Oncol. 2021; 22(6):801-12

24 Attal M, Richardson PG, Rajkumar SV, SanMiguel J, Beksac M, Spicka I, et al. Isatuximab plus pomalidomide and low-dose dexamethasone versus pomalidomide and low-dose dexamethasone in patients with relapsed and refractory multiple myeloma (ICARIA-MM): a randomised, multicentre, open-label, phase 3 study. Lancet. 2019;394(10214):2096-107.

25 San Miguel J, Weisel K, Moreau P, Lacy M, Song K, Delforge M, et al. Pomalidomide plus low-dose dexamethasone versus high-dose dexamethasone alone for patients with relapsed and refractory multiple myeloma (MM-003): a randomised, open-label, phase 3 trial. Lancet Oncol. 2013;14(11):1055-66.
26 Berenson JR, Cartmell A, Bessudo A, Lyons RM, Harb W, Tzachanis D, et al. CHAMPION-1: a phase $1 / 2$ study of once-weekly carfilzomib and dexamethasone for relapsed or refractory multiple myeloma. Blood. 2016; 30127(26):3360-8

27 Dimopoulos M, Quach H, Mateos MV, Landgren O, Leleu X, Siegel D, et al. Carfilzomib, dexamethasone, and daratumumab versus carfilzomib and dexamethasone for patients with relapsed or refractory multiple myeloma (CANDOR): results from a randomised, multicentre, open-label, phase 3 study. Lancet. 2020;396(10245):186-97.

28 Dimopoulos MA, Quach H, Mateos M-V, Landgren O, Leleu X, Siegel DS, et al. Carfilzomib, dexamethasone, and daratumumab versus carfilzomib and dexamethasone in relapsed or refractory multiple myeloma: updated efficacy and safety results of the phase 3 candor study. ASH Annual Meeting and Exposition 2020. Poster abstract 2325. Session: 653. Myeloma/amyloidosis: therapy, excluding transplantation: poster II, hematology disease topics \& pathways: therapies, combinations, clinically relevant, Sunday, $2020 \mathrm{Dec}$ 6, 7:00 AM-3:30 PM. 2020.

29 Moreau P, Dimopoulos MA, Mikhael J, Yong $\mathrm{K}$, Capra M, Facon T, et al. Isatuximab, carfilzomib, and dexamethasone in relapsed multiple myeloma (IKEMA): a multicentre, open-label, randomised phase 3 trial. Lancet. 2021;397(10292):2361-71.

30 Spencer A, Lentzsch S, Weisel K, Avet-Loiseau H, Mark TM, Spicka I, et al. Daratumumab plus bortezomib and dexamethasone versus bortezomib and dexamethasone in relapsed or refractory multiple myeloma: updated analysis of CASTOR. Haematologica. 2018 Dec;103(12):2079-87.

31 Gandhi UH, Cornell RF, Lakshman A, Gahvari ZJ, McGehee E, Jagosky MH, et al. Outcomes of patients with multiple myeloma refractory to CD38-targeted monoclonal antibody therapy. Leukemia. 2019 Sep;33(9): 2266-75.

32 Tzogani K, Penttilä K, Lähteenvuo J, Lapveteläinen T, Lopez Anglada L, Prieto C, et al. EMA review of belantamab mafodotin (blenrep) for the treatment of adult patients with relapsed/refractory multiple myeloma. Oncologist. 2021;26(1):70-6.

33 Lonial S, Lee HC, Badros A, Trudel S, Nooka AK, Chari A, et al. Belantamab mafodotin for relapsed or refractory multiple myeloma (DREAMM-2): a two-arm, randomised, open-label, phase 2 study. Lancet Oncol. 2019;21(2):207-21.

34 Chari A, Vogl DT, Gavriatopoulou M, Nooka AK, Yee AJ, Huff CA, et al. Oral selinexordexamethasone for triple-class refractory multiple myeloma. N Engl J Med. 2019;381: 727-38.

35 EMA. Nexpovio product information. 2021 [cited 2021 Aug 11]. Available from: https:// www.ema.europa.eu/en/medicines/human/ EPAR/nexpovio\#authorisation-details-section.
36 Kumar SK, Harrison SJ, Cavo M, de la Rubia J, Popat R, Gasparetto C, et al. Venetoclax or placebo in combination with bortezomib and dexamethasone in patients with relapsed or refractory multiple myeloma (BELLINI): a randomised, double-blind, multicentre, phase 3 trial. Lancet Oncol. 2020;21(12): 1630-42.

37 van de Donk N, Popat R, Larsen J, Minnema MC, Jagannath S, Oriol A, et al. First results of iberdomide (IBER; CC-220) in combination with dexamethasone (DEX) and daratumumab (DARA) or bortezomib (BORT) in patients with relapsed/refractory multiple myeloma (RRMM). ASH Annual Meeting and Exposition 2020. Abstract 724. Oral presentation. Session: 653. Myeloma/amyloidosis: therapy, excluding transplantation; novel approaches for relapsed/refractory myeloma and amyloidosis. Presented on Monday, 2020 Dec 7, 1:30 PM. 2020.

38 Richardson PG, Oriol A, Larocca A, Bladé J, Cavo M, Rodriguez-Otero P, et al. Melflufen and dexamethasone in heavily pretreated relapsed and refractory multiple myeloma. J Clin Oncol. 2021 Mar 1;39(7):757-67.

39 Bhutani M, Foureau DM, Atrash S, Voorhees PM, Usmani SZ. Extramedullary multiple myeloma. Leukemia. 2020;34:1-20.

40 D’Agostino M, Raje N. Anti-BCMA CAR Tcell therapy in multiple myeloma: can we do better? Leukemia. 2020;34:21-34.

41 Raje N, Berdeja J, Lin Y, Siegel D, Jagannath S, Madduri D, et al. Anti-BCMA CAR T-cell therapy bb2121 in relapsed or refractory multiple myeloma. N Engl J Med. 2019;380:172637.

42 Munshi NC, Anderson LD, Shah N, Jagannath S, Berdeja JG, Lonial S, et al. Idecabtagene vicleucel (ide-cel; bb2121), a BCMA-targeted CAR T-cell therapy, in patients with relapsed and refractory multiple myeloma (RRMM): Initial KarMMa results. Abstract 8503. Presented at ASCO 2020. Virtual meeting. J Clin Oncol. 2020 May 20;38(15_Suppl): 8503.

43 Berdeja JG, Krishnan AY, Oriol A, van de Donk NWCJ, Rodriguez-Otero P, Askari E, et al. Updated results of a phase 1, first-in-human study of talquetamab, a $\mathrm{G}$ protein-coupled receptor family $C$ group 5 member $D$ (GPRC5D) $\times$ CD3 bispecific antibody, in relapsed/refractory multiple myeloma (MM). J Clin Oncol. 2021;39(15_Suppl):8008.

44 Garfall A, Usmani SZ, Mateos MV, Nahi H, van de Donk NWCJ, San-Miguel JF, et al. Updated phase I results of teclistamab, a B-cell maturation antigen (BCMA) x CD3 bispecific antibody, in relapsed and/or refractory multiple myeloma (RRMM). Presented at the 2020 American Society of Hematology Annual Meeting, Abstract 180. Oral presentation. Session: 653. Myeloma/amyloidosis: therapy, excluding transplantation: novel therapies targeting B cell maturation antigen in relapsed/refractory multiple myeloma. Presented on Saturday, 2020 Dec 5, 12:45 PM. 2020. 